\title{
The Structure Optimization Design of Anti Re-entrainment Cone in Cyclone Separator
}

\author{
Zhiquan, Zuo ${ }^{1}$ \\ ${ }^{1}$ Changzhou University, \\ Changzhou, Jiangsu, 213016, China
}

\author{
Qidong Chen ${ }^{2}$ \\ ${ }^{2}$ Changshu Institute of Technology, \\ Changshu, Jiangsu, 215500, China
}

\begin{abstract}
In this study, the computational fluid dynamics (CFD) method was used to optimize the structure of anti reentrainment cone in cyclone separator. The Reynolds stress model (RSM) was used to simulate the flow fields inside a cyclone separator with different anti re-entrainment cone structure, and their axial and tangential velocity distribution. Based upon that, it could be concluded that an anti reentrainment cone with half ball surface located above the inlet of dust hopper and blow-down from dust hopper could lead to a smoother fluid field upon the inlet of the dust hopper. Besides, an appropriate effective section ratio and blow-down ratio of the cyclone studied in this article could be figured out.
\end{abstract}

Keywords-cyclone separator; anti re-entrainment cone; optimization design

\section{INTRODUCTION}

Gas cyclone separators are widely used in industries to separate dust from gas. How to increase the cyclone collection efficiency of nanoparticles is an emergency and important subject. Dust hopper, as a collector of particles, decreasing the particle re-entrainment from it can increase the cyclone collection efficiency. Until now, a considerable number of investigations [1-4] have been performed on dust hopper, but in these studies, downward and upward flow both occurred. Undoubtedly, the upward flow will increase the chance of particle escaping from dust.

The present study was undertaken in an effort to carry out a numerical study on the effect of different anti reentrainment cone structure and blow-down from dust hopper.

\section{MATHEMATICAL MODELS AND GEOMETRY}

Reynolds stress model (RSM) has been widely accepted as a promising numerical tool for solving the large-scale unsteady behaviour of complex turbulent flows. Encouraging results have been reported in recent literature and demonstrate the ability of RSM to capture the swirling flow instability and the energy containing coherent motion of such highly swirling flows. RSM methodology has been used in many articles to study the highly swirling flow in cyclone separators [5-7]. It will be used in this study to reveal the effect of different anti reentrainment cone structure and blow-down from dust hopper on the turbulent flow in the cyclone separator. Solver settings used in this study referred to [8].

The dimensions of the cyclone were displayed in Figure 1(a) and Table I. This cyclone was investigated experimentally by SM Fraser et al. [9]. In this study, a cone was located above the inlet of dust hopper. Its height was $0.04 \mathrm{~m}$, and the top surface diameter was $d_{c}$. The diameter of the coplanar section on the top surface of cone was $D_{c}$, and the flanks of cone and cyclone separator were parallel while the lower surface of cone and the inlet of dust hopper were coplanar. The dimensions of the cone were shown in Figure 1(b).Velocity inlet boundary condition was applied at inlet, outflow at gas outlet and blow-down surface of dust hopper and wall (no-slip) boundary condition at all other boundaries. The air inlet velocity $v_{\text {in }}$ equals $7.5 \mathrm{~m} / \mathrm{s}$.For the near-wall treatment, the standard wall function was used in the RSM simulation.

TABLE I. CONFIGURATION SIZE OF THE CYCLONE SEPARATOR M

\begin{tabular}{|c|c|c|c|c|c|c|c|c|c|}
\hline $\mathrm{D}$ & $\mathrm{a}$ & b & $\mathrm{d}_{\mathrm{e}}$ & $\mathrm{s}$ & $\mathrm{h}_{1}$ & $\mathrm{~h}_{2}$ & $\mathrm{~d}_{0}$ & $\mathrm{~d}_{\mathrm{h}}$ & $\mathrm{h}_{3}$ \\
\hline 0.19 & 0.038 & 0.095 & 0.064 & 0.095 & 0.285 & 0.475 & 0.0725 & 0.18 & 0.3 \\
\hline
\end{tabular}




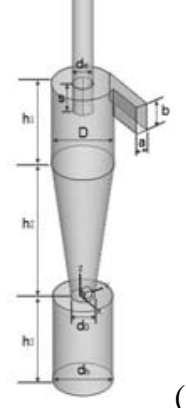

(a)

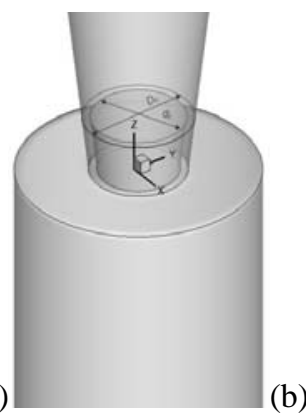

Figure 1. Schematic diagram for the cyclone geometry and coordinate definition

\section{RESULTS AND DISCUSSION}

\section{A. The velocity field of the cyclone with no blow-down}

For the purposes of discussion, the section between the cone and cyclone, which the air passed through, was defined as effective section, and Ratio was defined as effective section ratio $\left\{\right.$ Ratio $\left.=1-\left(d_{c} / D_{c}\right)^{2}\right\}$. Downward flow ratio was defined as the ratio of the mass quantity of inlet and downward flow at section $\mathrm{Z}=0.04 \mathrm{~m}$ $\left\{\right.$ downward flow radio $=Q_{m}($ down $) / Q_{m}($ in $\left.)\right\}$. The cyclone with different Ratio had been numerically investigated. The influence of the Ratio on the downward flow ratio was shown in Figure 2. From Figure 2, it could be clearly concluded that the downward flow ratio increased with Ratio. It meant that the mass quantity of the gas flowing into the dust hopper was growing. The downward flow ratio increased slowly at the Ratio range of $0.1 \sim 0.4$, linearly at $0.4 \sim 0.9$ and slowly at $0.9 \sim 1$. Five Ratios (Ratio= $0.2,0.4,0.6,0.8,1)$ were discussed in this study.

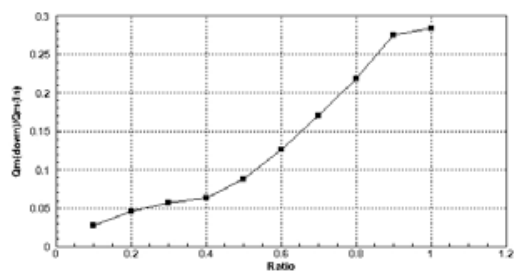

Figure 2. Influence of the Ratio on the downward flow ratio ( $Q_{m}$ (in) was the mass quantity of inlet, $Q_{m}($ down) was the mass quantity of downward flow at section $\mathrm{Z}=0.04 \mathrm{~m}$ )

Comparison of the time averaged tangential and axial velocity at section $\mathrm{Z}=0.36,0.635 \mathrm{~m}$ of five different Ratio cyclones were shown in Figure 3. Figure 3 showed that the anti re-entrainment cone had a limited effect on the distribution of the tangential velocity but on the axial velocity, especially in the cone part of the cyclone. Besides, the anti re-entrainment cone decreased the zone of downward flow in the cone part of the cyclone. This was not good for particles flowing into the dust hopper. In cylinder, the zone of downward flow changed little. Moreover, the anti re-entrainment cone reduced the axial velocity near the center and increased the time particles staying in cyclone, making particles have more time to separate from gas.

Figure 4 showed the zone (the gray zone in Figure 4) of downward flow at anti re-entrainment cone for different Ratio cyclones and the axial velocity distribution at section $\mathrm{Z}=0.035 \mathrm{~m}$. According to continuity equation, the blank near the wall in Figure 4 was the zone of upward flow. As could be seen from Figure 4, when ratio equalled 0.2 and 0.4 , the downward flow and upward flow divided the region between the anti re-entrainment cone and wall into two parts. This impeded the gas flowing into the dust hopper so seriously that the particles near the anti re-entrainment cone could not be brought into dust hopper smoothly by downward flow, increasing the chance particles escaping from the cyclone. When ratio equalled $0.6,0.8$ and 1, the gas motion could be seen as two regions with an outer region of downward vortex flow surrounding an inner region of upward vortex flow. The zone of downward flow and upward flow increased with Ratio. It could be concluded from the axial velocity distribution at section $\mathrm{Z}=0.035 \mathrm{~m}$ that two regions character reduced the region for downward flow, and downward flow and upward flow were so close that the space particles moving was limited. Because of that, the particles were taken out by the inner region of upward vortex flow easily. So, only an anti re-entrainment cone located above the inlet of the dust hopper could not improve the cyclone separation performance greatly.
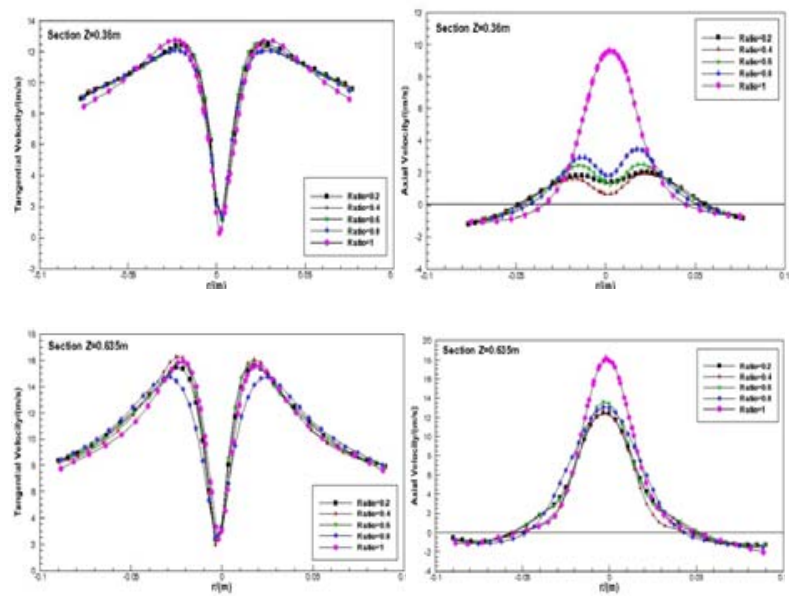

Figure 3. The radial profile for the time averaged tangential and axial velocity at different sections of different Ratio cyclones 


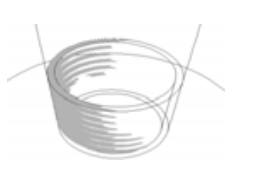

Ratio $=0.2$

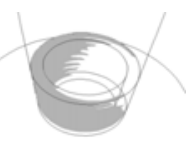

Ratio $=0.4$

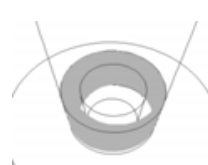

Ratio $=0.6$

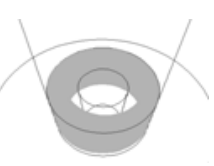

Ratio $=0.8$

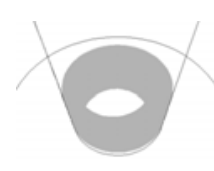

Ratio=1

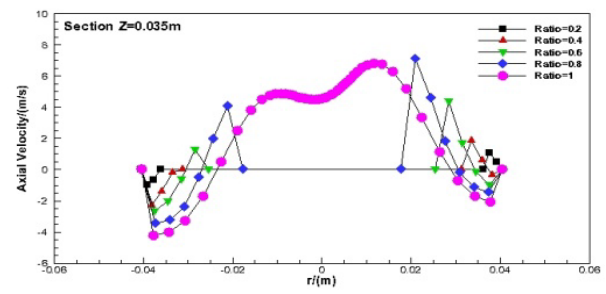

Figure 4. The zone of downward flow at anti re-entrainment cone for different Ratio cyclones and the axial velocity distribution at section $\mathrm{Z}=0.035 \mathrm{~m}$

\section{B. The velocity field of the cyclone with blow-down}

In order to make downward flow bring particles into the dust hopper successfully and prevent re-entrainment, the blow-down was applied to the dust hopper. Through multiple sets of test on blow-down ratio, it could be found that to make the downward flow fill with the zone between the anti re-entrainment cone and wall, the blowdown ratio equalled $10 \%$ when Ratio equalled 0.2 , $22 \%$ when Ratio equalled 0.4, 42\% when Ratio equalled 0.6, $75 \%$ when Ratio equalled 0.8 .

In Figure 5, the radial profiles for tangential and axial velocity of cyclones (Ratio $=0.2,0.4,0.6,0.8$ ) with blowdown ratio shown above were compared with that of the cyclone (Ratio=1) without blow-down. From Figure 5 it could be seen that the tangential velocity distributions were similar in the cone part of the five cyclones and the regions of the five downward flows were almost same in cylinder part. Because of blow-down, the axial velocity decreased sharply in cone part. The region of the downward flow in the cyclone which Ratio equalled 0.2 was the smallest, making particles flow into dust hopper hardly.

When the effective section was filled with downward flow, downward area ratio, which was the ratio of the area of downward region at one section and the area of this section, equalled Ratio. Decreasing the Ratio would decrease the quantity of the downward flow, and make particles flow into dust hopper hardly. Conversely, the blow-down ratio would increase, and it would set a great demand on the strainer in dust hopper or the dust collection bag, especially when a large amount of gas needed dealing with. Through calculation it could be figured out that, at section $\mathrm{Z}=0.04 \mathrm{~m}$, downward area ratio equalled 0.588 when Ratio equalled 1 , and that was similar to the downward area ratio when Ratio equalled 0.6 and blow-down ratio equalled $42 \%$. But another downward area ratio similar to 0.588 at the same section was the cyclone which Ratio equalled 0.4 and blow-down ratio equalled $22 \%$. Based on this, it could be figured out that the later blow-down ratio was $20 \%$ less than the earlier one, and the later cyclone was better.
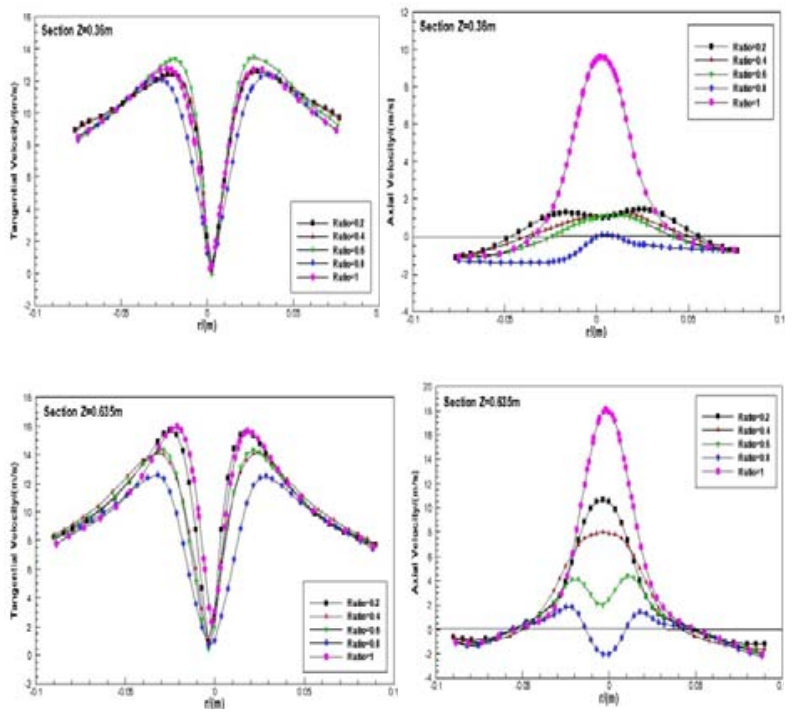

Figure 5. The radial profile for tangential and axial velocity at different sections for different Ratio(Ratio $=0.2,0.4,0.6,0.8,1)$ cyclone with different blow-down ratio(blow-down ratio=10\%,22\%,42\%,75\%,0)

On account of the problem, an improvement was made just as shown in Figure 6. The top surface of the anti reentrainment cone was changed into half ball surface. Through multiple sets of test on blow-down ratio, it could be found that to make the downward flow fill with the zone between the anti re-entrainment cone and wall, the blow-down ratio equalled $17 \%$ when Ratio equalled 0.4 . That was $5 \%$ less than the $22 \%$ before. The radial profiles for tangential and axial velocity at different sections for Ratio 0.4 and 1 cyclones with blow-down ratio17\% and 0 were compared as shown in Figure 7 . It could be concluded that the tangential velocity of Ratio 0.4 cyclone was larger than that of Ratio 1 cyclone in cone part and similar in cylinder part. Besides, the downward region and downward axial velocity distribution in the two cyclones were similar. The tangential velocity in the cyclone with improvement was larger than that in the cyclone without improvement, and the downward region and downward axial velocity also increased. That was better for particles flowing into dust hopper smoothly. 


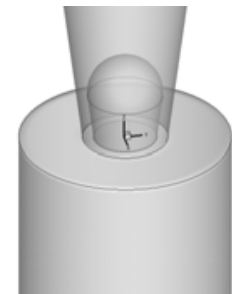

Figure 6. The improved anti re-entrainment cone geometry
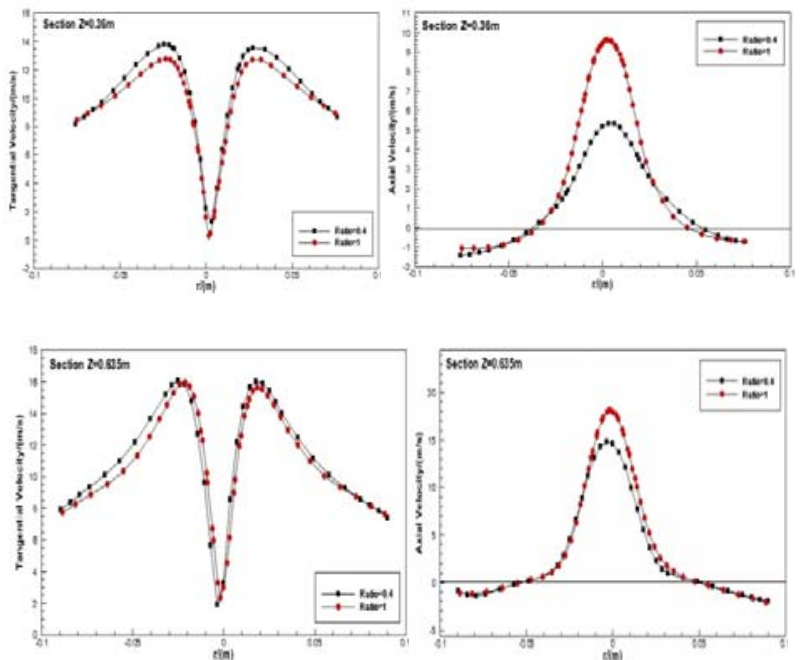

Figure 7. The radial profile for tangential and axial velocity at differen sections for different Ratio (Ratio $=0.4,1$ ) cyclone with different blowdown ratio(blow-down ratio=17\%,0)

\section{CONCLUSION}

In this paper, we numerically investigated the flow field inside cyclone separators with different anti reentrainment cone structure and blow-down ratio, and estimated the influences on the performance for particle separation. In this study, it could be concluded that only an anti re-entrainment cone located above the inlet of dust hopper could not avoid upward flow which would take particles out from dust hopper, but blow-down from dust hopper could avoid it and increase separation efficiency. Through the optimization design on the structure of anti re-entrainment cone in cyclone separator, it could be found that an anti re-entrainment cone with half ball surface would lead to a smoother velocity field.

\section{REFERENCES}

[1] Obermair S, Standinger G. The Effect of the Dust Outlet Geometry on the Separation Efficiency of a Gas Cyclone[C].// Nuremberg, Germany: 2001.1-8.

[2] Obermair S, Woisetschlager J, Standinger G. The Flow Pattern in the Dust Outlet Geometry of a Gas Cyclone and its Effects on Separation Efficiency[C].// Dusseldorf, Germany: 2001. 1-8.

[3] FU Shuang-cheng, SUN Guo-gang, GAO Cui-zhi, GUO Guang-jun. Numerical Simulation and Experiment Study of the Effect of Anti Re-entrainment Cone Locations on Cyclone Separator Performance [J].// 6th Annual Conference of Chinese Society of Particuology cum-symposium on Particle Technology across Taiwan Straits , 2008, 808-812. (In Chinese)

[4] ZHANG Jian, JIN You-hai. Numerical simulation of influence of blow-down on cyclone's separation performance [J]. // Journal of Engineering Design, 2008, 15(5), 313-318. (In Chinese)

[5] FENG Xi-ping, ZHAO Sheng-hai, LI Jin-xian, CAO Qi. Numerical simulation of swirling flow with different turbulent models [J]. // Journal of Aerospace Power, 2011, 26(6), 1209-1214. (In Chinese)

[6] Zhang ya, Liu shu-yan, Wang bao-guo. numerical simulation of the turbulence anisotropic behavior using the reynolds stress model in cyclone dust catcher[j].// journal of engineering thermophysics, 2005,26(plus),41-44.(in chinese)

[7] Mehdi Azadia, Mohsen Azadib, Ali Mohebbia. A CFD study of the effect of cyclone size on its performance parameters[J].// Journal of Hazardous Materials, 182(2010) 835-841.

[8] Chen Qidong, Zuo Zhiquan. Numerical analysis on different lateral inlet cyclone flow field [J]. // Journal of Engineering Science, 2014(2). (In Chinese).

[9] S M Fraser,A M Abdel-Razek and M Z Abdullah. Computational and experimental investigations in a cyclone dust separator [J]. //Journal of Process Mechanical Engineering, 1997 211-247. 